\title{
Análise epidemiológica de 9.897 óbitos por COVID-19 no período de março a maio de 2020, no Brasil
}

\section{Epidemiological analysis of 9,897 deaths from COVID-19 in the period from March to May 2020, in Brazil}

\author{
Daniele França dos Santos ${ }^{1}$ \\ Isabella Pereira Rosa de Castro ${ }^{2}$ (1) \\ Claudacy Pessoa Monte Santo ${ }^{3}$ [C]
}

1-4,6 Hospital Geral Roberto Santos (Salvador). Bahia, Brasil. nupefisio.hgrs@gmail.com, bellaprosa@hotmail.com, claudacyfisio@yahoo.com.br,
vivianepereiracorfis@gmail.com, carol.guerreiro@yahoo.com.br
${ }^{5}$ Autor para correspondência. Hospital Geral Roberto Santos (Salvador). Bahia, Brasil. fabiannafonseca@yahoo.com.br
Viviane Pereira Santos 4 (i) Fabianna Fonseca de Oliveira Figueiredo ${ }^{5}$ (1) Caroline Ferreira Guerreiro ${ }^{6}$ (1)
RESUMO | INTRODUÇÃO: O surgimento da pandemia de COVID-19 afetou severamente o mundo todo, inclusive o Brasil no que diz respeito ao número de óbitos. Dados epidemiológicos são importantes para o conhecimento do curso da doença, além de contribuir para realização de medidas preventivas, educacionais e técnicas. OBJETIVO: Analisar aspectos epidemiológicos dos óbitos por COVID-19 no período de março à maio de 2020 no Brasil. CASUÍsTICA E MÉTODOs: Trata-se de estudo descritivo, através de dados publicados e extraídos do Sistema de Informação do Ministério da Saúde entre março e maio de 2020. Analisaram-se frequências simples absoluta de 145.328 casos por COVID-19 e 9.89(6,8\%) óbitos. As análises incluíram faixa etária; grupo de risco de acordo com a faixa etária e distribuição geográfica. RESULTADOS: Os resultados mostraram que $69 \%$ tinham mais de 60 anos e $65 \%$ apresentavam pelo menos um fator de risco. A cardiopatia foi a principal comorbidade associada e esteve presente em 3.425 óbitos, seguida de diabetes, doença renal, doença neurológica e pneumopatia. A maioria dos óbitos foi registrada na região Sudeste seguida da Nordeste e Norte. CONCLUSÃo: As medidas de controle da infecção, de educação e orientação da população para o controle da COVID-19 devem ser intensificadas em indivíduos acima de 60 anos, com história de cardiopatias e em regiões de clima temperado.

PALAVRAS-CHAVE: COVID-19. Epidemiologia. Saúde pública.

\begin{abstract}
INTRODUCTION: The emergence of the COVID-19 pandemic has severely affected the whole world, including Brazil, concerning deaths. Epidemiological data are essential for understanding the course of the disease and contributing to preventive, educational, and technical measures. OBJECTIVE: To analyze epidemiological aspects of deaths due to COVID-19 from March to May 2020 in Brazil. CASUISTICS AND METHODS: This is a descriptive study using data published and extracted from the Ministry of Health's Information System between March and May 2020. Absolute simple frequencies of 145,328 cases were analyzed by COVID-19 and $9.89(6,8 \%)$ deaths. The analyzes included age group; risk group according to age and geographic distribution. RESULTS: The results showed that $69 \%$ were over 60 years old, and $65 \%$ had at least one risk factor. Heart disease was the main associated comorbidity and was present in 3,425 deaths, followed by diabetes, kidney disease, neurological disease, and pneumopathy. Most deaths were registered in the Southeast, followed by the Northeast and North. CONCLUSION: Infection control, education, and population orientation measures for the control of COVID-19 should be intensified in individuals over 60 years of age, with a history of heart disease, and in temperate regions.
\end{abstract}

KEYWORDS: COVID-19. Epidemiology. Public health. 


\section{Introdução}

O novo coronavírus (SARS-CoV-2) que surgiu em Wuhan, China, no final de 2019 se espalhou rapidamente para todas as províncias chinesas ${ }^{1}$, e em 31 de dezembro o governo chinês notificou a Organização Municipal de Saúde (OMS) sobre os casos de pneumonia com etiologia desconhecida. ${ }^{2}$ Em sete de janeiro de 2020, autoridades chinesas identificaram um novo tipo de coronavírus isolado, e no dia 12 a China compartilhou a sequência genética do SARS-CoV-2. Em 12 de março de 2020, a OMS declarou o surto global de COVID-19 uma pandemia com a confirmação de 125.048 casos e 4.613 óbitos. 2 Entretanto, a pesquisa do Centro de Controle e Prevenção de Doenças da China (CCDC) afirma que cerca de $80 \%$ das novas infecções por SARS-CoV-2 são classificadas como leves, $14 \%$ como graves e apenas $5 \%$ como críticas, o que inclui quadro de insuficiência respiratória, falência múltipla dos órgãos e sepse. . $^{-}$

No Brasil, o número de casos de COVID-19 tem crescido rapidamente, assim como os registros de óbitos. O primeiro caso de COVID-19 no Brasil foi confirmado no estado de São Paulo, no dia 26 de fevereiro, em um brasileiro com 61 anos de idade que tinha realizado uma viagem para Itália, onde houve um aumento expressivo de casos da doença. ${ }^{4}$ Desde então, esforços para conter o vírus estão em andamento ${ }^{5}$; no entanto, dadas as muitas incertezas em relação à transmissibilidade e virulência de patógenos, a eficácia desses esforços ainda é desconhecida. $\underline{6}$

Dados epidemiológicos são importantes para o conhecimento do problema, além de contribuir na realização de medidas preventivas, educacionais e

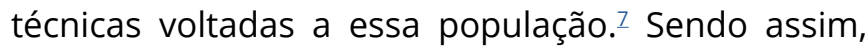
informações sobre as características epidemiológicas da COVID-19 são necessárias para a criação de estratégias de prevenção e controle do avanço da doença nos países afetados pelo vírus. Neste estudo, foi realizada uma descrição das características epidemiológicas dos óbitos por COVID-19 no Brasil, no período de dezessete de março a oito de maio de 2020, de acordo com a faixa etária, grupos de risco de acordo com a faixa etária e distribuição de casos por regiões e Unidades Federativas do país.
Trata-se de um estudo epidemiológico, descritivo e retrospectivo, com abordagem quantitativa da população com diagnóstico de COVID-19 no Brasil. Os dados foram obtidos diretamente do Sistema de Informação do Ministério da Saúde (boletim epidemiológico, 2020), acessados no período de 17 março a oito de maio de 2020, abrangendo faixa etária, grupo de risco de acordo com a faixa etária e distribuição geográfica dos óbitos. ${ }^{8}$

Para a descrição do perfil epidemiológico dos casos confirmados das variáveis utilizadas para a pesquisa, como faixa etária e fatores de risco, foram utilizadas informações compostas nestes boletins organizadas em tabelas.

Para cálculo da taxa de letalidade da COVID-19, utilizou-se o número de óbitos por COVID-19 detectados em pessoas das regiões e Unidade Federativa (UF) e foi realizada a divisão pelo número total da população infectada multiplicada por cem.

A população do estudo incluiu indivíduos independentes da faixa etária, com diagnóstico de COVID-19 no Brasil, que foram a óbito no período analisado. Foram excluídos os óbitos que aguardavam a confirmação da causa. As variáveis de desfecho consideradas foram as taxas de mortalidade por COVID-19 no período de 52 dias.

O Boletim Epidemiológico, editado pela Secretaria de Vigilância em Saúde do Brasil, é uma publicação de caráter técnico-científico, acesso livre, formato eletrônico com periodicidade diária e semanal para os casos de monitoramento e investigação de doenças específicas sazonais. Ele se configura como instrumento de vigilância para promover a disseminação de informações relevantes qualificadas, com potencial para contribuir com a orientação de ações em Saúde Pública no país. 4

Os dados obtidos através do Sistema de Informação do Ministério da Saúde foram tabulados e analisados através de estatística descritiva no Microsoft Excel, versão 2003, e apresentados através de tabelas, sumarizando as características e distribuição dos valores observados. As variáveis categóricas foram apresentadas em termos absolutos e/ou frequência relativa percentual. 
Os dados utilizados estão disponíveis na internet para consulta livre na forma de dados agregados por municípios, ou seja, as mesmas não foram coletadas de maneira individualizada e nominal. Nesse sentido, não há possibilidade de dano de ordem física ou moral na perspectiva do indivíduo e das coletividades, por terem sidos respeitados os princípios contidos na Resolução 466, de 12 de dezembro de 2012. ${ }^{9}$ Desse modo, o presente artigo não demandou submissão ao Comitê de Ética em Pesquisa.

\section{Resultados}

Até o dia oito de maio de 2020, foram confirmados 145.328 casos por COVID-19 no Brasil, deste total, 9.897(6,8\%) foram a óbito.

Entre os óbitos confirmados por COVID-19, 69\% tinham mais de 60 anos (tabela 1) e 65\% apresentavam pelo menos um fator de risco. A cardiopatia foi a principal comorbidade associada e esteve presente em 3.425 dos óbitos, seguida de diabetes (em 2.660 óbitos), doença renal (621), doença neurológica (550) e pneumopatia (544). Em todos os grupos de risco, a maioria dos indivíduos tinha 60 anos ou mais, exceto para obesidade. A distribuição dos óbitos de acordo com o grupo de risco e a faixa etária estão descritos na tabela 2.

A maior parte dos casos concentrou-se na região Sudeste com $64.756(44,6 \%)$ seguido das regiões Nordeste com $45.724(31,5 \%)$ casos e Norte com $23.207(16 \%)$ casos. Em relação aos óbitos, os Estados que apresentaram os maiores números foram São Paulo 3.416, Rio de Janeiro 1503, Ceará 966, Pernambuco 927 e Amazonas 874. Uma distribuição espacial dos óbitos confirmados pela doença está mostrada na tabela 3.

Tabela 1. 9.897 Óbitos por COVID-19 segundo a faixa etária. Brasil, 2020

\begin{tabular}{ll}
\hline Varíavel & $\mathrm{N}(\%)$ \\
\hline Faixa Etária & \\
\hline$<1$ (anos) & $12(0.14)$ \\
1 a 5 (anos) & $6(0.07)$ \\
6 a19 (anos) & $33(0.40)$ \\
20 a 29 (anos) & $108(1.31)$ \\
30 a 39 (anos) & $388(4.71)$ \\
40 a 49 (anos) & $708(8.59)$ \\
50 a 59 (anos) & $1281(15.5)$ \\
60 a 69 (anos) & $1903(23.11)$ \\
70 a 79 (anos) & $1959(23.79)$ \\
80 a 89 (anos) & $1439(17.47)$ \\
90 ou mais (anos) & $396(4.8)$ \\
\hline
\end{tabular}

Fonte: Secretaria de Vigilância em Saúde/ Ministério da Saúde. Dados até o dia 08 de maio de 2020.

Tabela 2. 9.897 Óbitos por COVID-19 classificados por grupos de risco e faixa etária. Brasil, 2020

\begin{tabular}{lll}
\hline Varíavel & $\begin{array}{l}<0 \text { anos } \\
\mathrm{N}(\%)\end{array}$ & $\begin{array}{l}60 \text { ou mais anos } \\
\mathrm{N}(\%)\end{array}$ \\
\hline Grupos de Risco & & \\
\hline Cardiopatia & $2.774(81)$ & $651(19)$ \\
Diabetes & $2.001(75)$ & $659(25)$ \\
Doença Renal & $480(77)$ & $141(23)$ \\
Doença Neurológica & $490(89)$ & $60(11)$ \\
Pneumopatia & $455(84)$ & $89(16)$ \\
Obesidade & $153(43)$ & $202(57)$ \\
Imunodepressão & $244(65)$ & $133(35)$ \\
Asma & $127(65)$ & $68(35)$ \\
\hline
\end{tabular}

Fonte: Secretaria de Vigilância em Saúde/ Ministério da Saúde. Dados até o dia 08 de maio de 2020. 
Tabela 3. Distribuição de casos de óbito por COVID-19 por região e Unidade da Federação. Brasil, 2020

\begin{tabular}{|c|c|c|c|}
\hline Variáveis & $\begin{array}{l}\text { Casos confirmados } \\
\mathrm{N}=145.328\end{array}$ & $\begin{array}{l}\text { Óbitos } \\
N=9.897\end{array}$ & $\begin{array}{ll}\text { Taxa } & \text { de } \\
\text { Letalidade } & \\
\end{array}$ \\
\hline UF/Região & $\mathbf{N}(\%)$ & $\mathbf{N}(\%)$ * & \\
\hline \multicolumn{4}{|l|}{ Norte } \\
\hline Rondônia & $1.222(0.84)$ & $39(0.39)$ & 3,19 \\
\hline Acre & $1.177(0.80)$ & $38(0.38)$ & 3,22 \\
\hline Amazonas & $10.727(7.38)$ & $874(8.81)$ & 8,14 \\
\hline Roraima & $1.124(0.77)$ & $39(0.39)$ & 3,46 \\
\hline Pará & $6.141(4.22)$ & $515(5.19)$ & 8,38 \\
\hline Amapá & $2.322(1.59)$ & $66(0.66)$ & 2,84 \\
\hline Tocantins & $494(0.33)$ & $9(0.09)$ & 1,82 \\
\hline \multicolumn{4}{|l|}{ Nordeste } \\
\hline Maranhão & $5.909(4.06)$ & $330(3.32)$ & 5,58 \\
\hline Piauí & $1.131(0.77)$ & $37(0.37)$ & 3,3 \\
\hline Ceará & $14.956(10.29)$ & $966(9.73)$ & 6,4 \\
\hline Rio Grande do Norte & $1.821(1.25)$ & $81(0.81)$ & 4,4 \\
\hline Paraíba & 2.031 (1.39) & $114(1.14)$ & 5,6 \\
\hline Pernambuco & $11.587(7.97)$ & $927(9.34)$ & 8,0 \\
\hline Alagoas & $2.033(1.39)$ & $108(1.08)$ & 5,3 \\
\hline Sergipe & $1.438(0.98)$ & $28(0.28)$ & 1,9 \\
\hline Bahia & $4.818(3.31)$ & $183(1.84)$ & 3,8 \\
\hline \multicolumn{4}{|l|}{ Sudeste } \\
\hline Minas Gerais & $2.943(2.02)$ & $111(1.11)$ & 3,8 \\
\hline Espírito Santo & $4.242(2.91)$ & $165(1.66)$ & 3,9 \\
\hline Rio de Janeiro & $15.741(10.83)$ & $1.503(15.15)$ & 9,5 \\
\hline São Paulo & $41.830(28.78)$ & $3.416(34.43)$ & 8,16 \\
\hline \multicolumn{4}{|l|}{ Centro-Oeste } \\
\hline Mato Grosso do Sul & $326(0.22)$ & $11(0.11)$ & 3,4 \\
\hline Mato Grosso & $457(0.31)$ & $14(0.14)$ & 3,1 \\
\hline Goiás & $1.053(0.72)$ & $49(0.49)$ & 4,6 \\
\hline Distrito Federal & $2.442(1.68)$ & $37(0.37)$ & 1,5 \\
\hline \multicolumn{4}{|l|}{ Sul } \\
\hline Paraná & $1.711(1.17)$ & $106(1.06)$ & 6,2 \\
\hline Santa Catarina & $3.205(2.20)$ & $63(0.63)$ & 1,9 \\
\hline Rio Grande do Sul & $2.447(1.68)$ & $91(0.91)$ & 3,7 \\
\hline
\end{tabular}

Fonte: Secretaria de Vigilância em Saúde/ Ministério da Saúde. Dados até o dia 08 de maio de 2020.

\section{Discussão}

Desde o início da epidemia, a partir da cidade de Wuhan, na China, a disseminação da doença ocorreu em curto espaço de tempo, deixando de ser um evento local para uma pandemia global em menos de quatro meses. ${ }^{1}$ Como o SARS-CoV-2 pode causar infecções letais, uma análise de casos fatais tem sido uma prioridade da saúde pública no mundo. O presente estudo analisa casos fatais disponíveis em registros públicos durante a pandemia da COVID-19 no Brasil de dezessete de março a oito de maio de 2020, quando um total de 9.897 óbitos foram identificados.

Apesar da tendência crescente de novos casos diários no estágio inicial, esse número na China tem sido menor do que fora desde 26 de fevereiro de 2020 . ${ }^{10}$ Segundo dados internacionais, até oito de maio de 2020 foram confirmados 4.006.555 casos de COVID-19, com 275.755 óbitos. ${ }^{-}$O Brasil, neste mesmo período, estava como oitavo em número de casos confirmados e o décimo primeiro em número de óbitos. ${ }^{8}$ Apesar das medidas restritivas de isolamento social impostas pelas autoridades de saúde em todo o mundo na tentativa de retardar a propagação do vírus, o número de infectados continua alto, assim como o número de mortes causadas pela doença. ${ }^{11}$ 
De acordo com o Centro Chinês de Controle e Prevenção de Doenças, $81 \%$ dos pacientes com COVID-19 tiveram um caso leve, e $87 \%$ tinham entre 30 e 79 anos. 10 A taxa de mortalidade de casos foi maior naqueles com casos críticos (49\%) e pacientes com idade igual ou superior a 80 anos (14,8\%). .10 No presente estudo, os grupos entre 60-69 e o de 7079 anos apresentaram um maior número de óbitos. Aproximadamente $69 \%$ dos casos confirmados de óbito por COVID-19 no Brasil têm idade igual ou superior a 60 anos. Adicionalmente, idosos são considerados um fator de preocupação para a contaminação com o SARS-CoV-2, uma vez que o aumento da idade está associado à morte. $\underline{12}$

No Brasil, de acordo com este estudo, a cardiopatia foi a principal comorbidade associada à mortalidade pelo vírus, sugerindo um envolvimento cardíaco direto pelo vírus. Sabe-se que as complicações cardíacas, como insuficiência cardíaca, arritmia ou infarto agudo do miocárdio (IAM) são comuns em pacientes com pneumonia, principalmente em indivíduos com idade avançada, doenças cardiovasculares prévias e maior gravidade da pneumonia no momento da admissão hospitalar. A literatura descreve ainda que a doença cardíaca coronariana está associada a eventos agudos e a desfechos negativos em infecções virais respiratórias, o que tem sido explicado por respostas sistêmicas e citocinas pró-inflamatórias, que são mediadores da aterosclerose e contribuem para a ruptura de placas devido a inflamação local e alterações hemodinâmicas, que predispõem à isquemia e trombose. $\frac{12}{\underline{12}}$

Uma meta-análise das comorbidades sugeriu que a hipertensão foi prevalente em aproximadamente $17 \%$ dos pacientes, e diabetes, doenças cardiovasculares e doenças do sistema respiratório estavam presentes em $8 \%, 5 \%$ e $2 \%$ dos casos, respectivamente. 13 Nosso estudo também mostrou que doenças cardiovasculares, diabetes e doenças respiratórias foram fatores associados aos óbitos por COVID-19. O estudo Souza, Leal e Santos apontou para uma progressão mais rápida da COVID-19 em quem possui comorbidades cardiovasculares, com uma média de dias do início dos primeiros sintomas ao óbito inferior em quase quatro dias (3,9 dias na média e 4,0 na mediana) quando comparados o grupo que possuía enfermidades cardiovasculares prévias e o grupo controle. Este processo decorre dos efeitos do SARS-CoV-2 no organismo humano, como a ligação do vírus à enzima conversora de angiotensina 2 (ECA2) encontrada nas superfícies das células cardíacas, renais e pulmonares. ${ }^{14}$ Portanto, a presença de comorbidades cardiovasculares acelera a mortalidade por COVID-19.

Mertz et al. $\frac{15}{}$ publicaram um estudo sobre a influenza e sugeriram que, comparado a pacientes sem comorbidades, o risco de morte para pacientes graves ocorre com mais frequência naqueles que apresentaram doença pulmonar obstrutiva crônica (DPOC) e que tinham doença cardiovascular. Nos dados do nosso país, no geral, os pacientes graves eram mais velhos e tinham um número mais significativo de condições comórbidas do que aqueles não graves, entre elas também pneumopatias, sugerindo que idade e comorbidades podem ser fatores de risco para pacientes críticos.

Doenças como hipertensão, DM, disfunções respiratórias, cardiovasculares e suas condições de suscetibilidade podem estar ligadas à patogênese do COVID-19. As doenças crônicas compartilham várias características semelhantes aos distúrbios infecciosos, como o estado pró-inflamatório e a atenuação da resposta imune inata. Além disso, distúrbios metabólicos podem levar à baixa função imune, o que pode tornar os indivíduos mais suscetíveis a complicações da doença. $\frac{13}{}$

No Brasil, de acordo com o presente estudo, a maior parte dos casos concentrou-se na região Sudeste, seguido das regiões Nordeste, Sul, Centro-Oeste e Norte. As maiores taxas de letalidade foram registradas no Sudeste, seguido de Nordeste, Norte, CentroOeste e Sul. Uma hipótese é que regiões que possuem os maiores centros rodoviários, onde há grande fluxo de pessoas, são os principais eixos de disseminação da doença; além disso, municípios podem apresentar maior vulnerabilidade aos efeitos da doença em virtude da maior proporção de idosos..$^{16}$

Devido às dimensões continentais do Brasil, estendendo-se por áreas temperadas, subtropicais e equatoriais, é possível identificar distintos padrões de sazonalidade do vírus influenza nas diferentes regiões do país. ${ }^{11}$ Nos países de clima temperado, o clima seco e frio é uma condição importante para o início da epidemia, favorecendo tanto a capacidade do vírus de sobreviver e se espalhar, quanto a depressão do sistema imunológico de seu hospedeiro. ${ }^{17}$ Essa informação traz uma compreensão da sazonalidade da influenza em áreas tropicais e pode ser usada para propor estratégias de prevenção e controle mais eficazes. 
O presente estudo mostra que São Paulo e Rio de Janeiro foram os estados que apresentaram um maior número de óbitos. Este fato pode estar associado ao fato de que esses Estados somam uma população de 63 milhões de habitantes, $29,7 \%$ da população brasileira. ${ }^{17}$ Além disso, são os locais onde foram registrados os primeiros casos de óbitos pela COVID-19 no Brasil, embora não seja possível afirmar se foram as portas de entrada da doença no país. ${ }^{19}$

É necessário destacar ainda que a taxa de letalidade também é influenciada pela subnotificação da doença e de óbitos..$^{18}$ Estados com pouca testagem e nos quais os testes são direcionados apenas para pacientes graves tendem a gerar taxas de letalidade maiores, pois não consideram o número real de doentes. ${ }^{19} \mathrm{~A}$ diferença entre a taxa mais alta de casos confirmados de COVID 19 (29\% São Paulo) e a mais baixa $(0,22 \%$ Mato Grosso do Sul) sugere que possuem vigência políticas distintas para a realização de testes e notificação.

Leung ${ }^{20}$ sugeriu que a taxa de mortalidade pode estar associada ao atraso da hospitalização, conforme implicado no tempo médio desde o primeiro sintoma até a internação. Vale ressaltar que a maioria dos casos incluídos no seu estudo ocorreu antes do anúncio do governo chinês sobre a preocupação da saúde pública com a pneumonia, bem como com a possível transmissão de homem para homem. Além disso, tratava-se de um período de ocorrência habitual de gripe na região, portanto a população ainda não estava ciente da necessidade de hospitalização.

A mortalidade é a questão mais importante ao lidar com o surto inesperado ou esperado e estabelecer prioridades para controlar as epidemias. Em circunstâncias de surto, a manutenção do sistema de saúde é o ponto-chave, especialmente quando há um aumento significativo no número de casos confirmados na comunidade, como aconteceu no Brasil e em outros países do mundo.

$\mathrm{Na}$ tentativa de gerenciar esse desafio, medidas de controle de fronteiras, realocação de recursos, identificação de casos e quarentena dos casos suspeitos, controle da distribuição de materiais de proteção individual (EPI) e educação da população quanto aos hábitos de higiene das mãos têm sido conduzidas no país, atenuando os efeitos da doença quando comparada a outras regiões.

O presente estudo possui algumas limitações. A taxa de mortalidade hospitalar relatada no presente estudo considerou apenas casos hospitalizados obtidos nas páginas oficiais das autoridades de saúde brasileiras. Alguns pacientes podem não ter sido hospitalizados devido à falta de recursos e outros ainda não terem sido diagnosticados por insuficiência de testes disponíveis em algumas regiões. Consequentemente, a taxa real de mortalidade de casos pode ser muito maior que a taxa de mortalidade hospitalar.

\section{Considerações finais}

As medidas de controle da infecção, de educação e orientação da população para o controle da COVID-19 devem ser intensificadas em indivíduos acima de 60 anos, com história de cardiopatias e em regiões de clima temperado.

Esta análise preliminar mostra os achados epidemiológicos iniciais da COVID-19, mas o monitoramento contínuo de pacientes com esta doença ainda se faz necessário.

\section{Contribuições dos autores}

Todos os autores foram responsáveis pela concepção e desenho do estudo. Santos DF, Castro IPR e Guerreiro CF realizaram a análise dos dados, interpretação dos resultados e escreveram o manuscrito. Santos VP, Santo CPM e Figueiredo FFO contribuíram com o conteúdo intelectual crítico e revisão final do artigo.

\section{Conflitos de interesses}

Nenhum conflito financeiro, legal ou político envolvendo terceiros (governo, empresas e fundações privadas, etc.) foi declarado para nenhum aspecto do trabalho submetido (incluindo, mas não se limitando a subvenções e financiamentos, participação em conselho consultivo, desenho de estudo, preparação de manuscrito, análise estatística, etc.). 


\section{Referências}

1. Comissão Municipal de Saúde Wuhan (CHN). Comissão Municipal de Saúde, Relatório da pneumonia na cidade de Wuhan. Wuhan: Comissão Municipal de Saúde Wuhan; 2019.

2. World Health Organization (WHO). Coronavirus disease (COVID-19) pandemic [Internet]. Geneva: World Health Organization; 2020. Disponível em: https://www.who.int/ emergencies/diseases/novel-coronavirus-2019

3. National Health Commission of the People's Republic of China (CHN). Update on the novel coronavirus pneumonia outbreak. Beijing, China: National Health Commission of the People's Republic of China; 2020. Disponível em: http://en.nhc.gov. cn/2020-02/13/c 76512.htm

4. Ministério da saúde (Brasil), Secretaria de Vigilância em Saúde. Boletim epidemiológico: Doença pelo coronavírus 2019. [Internet]. Brasília: Ministério da saúde. 2020. Disponível em: https://www. ghc.com.br/files/be6.pdf

5. Decreto $n^{\circ}$ 64.887, de 26 de março de 2020 (Brasil). Institui decretos do Governo de SP com medidas de prevenção e combate ao novo coronavírus. [Internet]. Diário Oficial. 2020. Disponível em: https://www.al.sp.gov.br/norma/193382

6. Li R, Pei S, Chen B, Yimeng C, Zhang T, Yang W, et al. Substantial undocumented infection facilitates the rapid dissemination of novel coronavirus (SARS-CoV2). Science. 2020;368(6490):489-93. https://doi.org/10.1126/science.abb3221

7. Lima-Costa MF, Barreto SM. Tipos de estudos epidemiológicos: conceitos básicos e aplicações na área do envelhecimento. Epidemiol. Serv. Saúde. 2003;12(4):189-201. http://dx.doi. org/10.5123/S1679-49742003000400003

8. Ministério da Saúde (Brasil), Secretaria de Vigilância em Saúde. Boletim Epidemiológico Especial: COE -COVID19. [Internet]. Brasília: Ministério da Saúde. 2020. Disponível em: https:// portalarquivos.saude.gov.br/images/pdf/2020/May/09/2020-0506-BEE15-Boletim-do-COE.pdf

9. Resolução n o 466, de 12 de dezembro de 2012 (Brasil). Aprova diretrizes e normas regulamentadoras de pesquisas envolvendo seres humanos. [Internet]. Brasília: Conselho Nacional de Saúde. 2012 [citado 2014 Mar 11]. Disponível em: https://conselho.saude. gov.br/resolucoes/2012/Reso466.pdf

10. Lai CC, Wang CY, Wang YH, Hsueh SC, Ko WC, Hsueh PR. Global epidemiology of coronavirus disease 2019 (COVID-19): disease incidence, daily cumulative index, mortality, and their association with country healthcare resources and economic status. Int J Antimicrob Agents. 2020;55(4):105946. https://doi.org/10.1016/j. ijantimicag.2020.105946
11. Melo GC, Duprat IP, Araújo KCGM, Fischer FM, Araújo Neto RA. Projeção da taxa cumulativa de óbitos por COVID-19 no Brasil: um estudo de modelagem. Rev. Bras. epidemiol. 2020;23:e200081. https://doi.org/10.1590/1980-549720200081

12. Zhou F, Yu T, Du R, Fan G, Liu Y, Liu Z, et al. Clinical course and risk factors for mortality of adult inpatients with COVID-19 in Wuhan, China: a retrospective cohort study. Lancet. 2020;395(10229):1054-62. https://doi.org/10.1016/s0140$\underline{6736(20) 30566-3}$

13. Yang J, Zheng Y, Gou X, Pu K, Chen Z, Guo Q, et. al. Prevalence of comorbidities in the novel Wuhan coronavirus (COVID-19) infection: a systematic review and meta-analysis. Int J Infect Dis. 2020;94:91-5. https://doi.org/10.1016/j.jijid.2020.03.017

14. Souza CDF, Leal TC, Santos LG. A Existência Prévia de Doenças do Aparelho Circulatório Acelera a Mortalidade por COVID-19?. Arq. Bras. Cardiol. 2020;115(1):146-7. https://doi.org/10.36660/ abc.20200486

15. Mertz D, Kim TH, Johnstone J, Lam PP, Science M, Kuster SP, et al. Populations at risk for severe or complicated influenza illness: systematic review and meta-analysis. BMJ. 2013;347(3):f5061. https://doi.org/10.1136/bmj.f5061

16. Barbosa IR, Galvão MHR, Souza TA, Gomes SM, Medeiros AA, Lima KC. Incidência e mortalidade por Covid-19 na população idosa brasileira e sua realação com indicadores contextuais: um estudo ecológico. Rev. Bras. Geriatr. Gerontol. 2020;23(1):e200171. https://doi.org/10.1590/1981$\underline{22562020023.200171}$

17. Almeida ARM. Dinâmica sazonal da influenza no Brasil: a importância da latitude e do clima. [dissertação] [Internet]. Rio de Janeiro: Fundação Oswaldo Cruz. Escola Nacional de Saúde Pública Sergio Arouca; 2018. Disponível em: https://www.arca.fiocruz.br/bitstream/icict/34080/2/ve_ Alexandra_Ribeiro_ENSP_2018

18. Ministério da Saúde (Brasil), Centro de Operações de Emergência em Saúde Pública. Boletim COE COVID-19: Situação epidemiológica-Doença pelo coronavírus 2019. [Internet]. Brasília: Ministério da Saúde. 2019. Disponível em: https:// portalarquivos.saude.gov.br/images/pdf/2020/April/21/BE13--Boletim-do-COE.pdf

19. Souza CD, Paiva JPS, Leal TC, Silva LF, Santos LG. Evolução espaçotemporal da letalidade por COVID-19 no Brasil, 2020. J. bras. pneumol. 2020;46(4): e20200208. https://doi. org/10.36416/1806-3756/e20200208

20. Leung C. Clinical features of deaths in the novel coronavirus epidemic in China. Rev. Med. Virol. 2020;30(3):1-4. https://doi. org/10.1002/rmv.2103 